\title{
Development and analysis of a real-time system for automated detection of improvised explosive device indicators from ground vehicles
}

Citation for published version (APA):

van de Wouw, D. W. J. M., ter Haar, F. B., Dubbelman, G., \& de With, P. H. N. (2019). Development and analysis of a real-time system for automated detection of improvised explosive device indicators from ground vehicles. Journal of Electronic Imaging, 28(4), [043009]. https://doi.org/10.1117/1.JEl.28.4.043009

DOI:

10.1117/1.JEl.28.4.043009

Document status and date:

Published: 01/07/2019

Document Version:

Publisher's PDF, also known as Version of Record (includes final page, issue and volume numbers)

Please check the document version of this publication:

- A submitted manuscript is the version of the article upon submission and before peer-review. There can be important differences between the submitted version and the official published version of record. People interested in the research are advised to contact the author for the final version of the publication, or visit the $\mathrm{DOI}$ to the publisher's website.

- The final author version and the galley proof are versions of the publication after peer review.

- The final published version features the final layout of the paper including the volume, issue and page numbers.

Link to publication

\footnotetext{
General rights

- You may freely distribute the URL identifying the publication in the public portal. follow below link for the End User Agreement:

www.tue.nl/taverne

Take down policy

If you believe that this document breaches copyright please contact us at:

openaccess@tue.nl

providing details and we will investigate your claim.
}

Copyright and moral rights for the publications made accessible in the public portal are retained by the authors and/or other copyright owners and it is a condition of accessing publications that users recognise and abide by the legal requirements associated with these rights.

- Users may download and print one copy of any publication from the public portal for the purpose of private study or research.

- You may not further distribute the material or use it for any profit-making activity or commercial gain

If the publication is distributed under the terms of Article 25fa of the Dutch Copyright Act, indicated by the "Taverne" license above, please 


\section{Electronnic Imaging}

\section{Development and analysis of a real- time system for automated detection of improvised explosive device indicators from ground vehicles}

Dennis W. J. M. van de Wouw

Frank B. ter Haar

Gijs Dubbelman

Peter H. N. de With 


\title{
Development and analysis of a real-time system for automated detection of improvised explosive device indicators from ground vehicles
}

\author{
Dennis W. J. M. van de Wouw, ${ }^{a, b, *}$ Frank B. ter Haar, ${ }^{c}$ Gijs Dubbelman, ${ }^{a}$ and Peter H. N. de With ${ }^{a}$ \\ ${ }^{a}$ Eindhoven University of Technology, Faculty of Electrical Engineering, SPS-VCA, Eindhoven, The Netherlands \\ ${ }^{b}$ ViNotion B.V., Eindhoven, The Netherlands \\ 'TNO, Den Haag, The Netherlands
}

\begin{abstract}
We propose a real-time change detection system to be used as a vehicle-mounted early-warning system for indicators of improvised explosive devices. Within the context of military route clearance, the system automatically detects suspicious changes in the environment with respect to a previous patrol. For this purpose, historical images of the live scene are retrieved from a database and registered to the live image through 2.5-D view synthesis, using the three-dimensional (3-D) scene geometry acquired from a stereo camera. Changes are then found using local-area statistics in the CIE-Lab color space. A set of spatiotemporal filters is used to reject irrelevant alarms, resulting in a limited set of confident changes to be presented to the operator through an interactive graphical user interface. Next to the algorithmic contributions, we elaborate on the real-time design, featuring graphical processing units for the most time-consuming processing tasks, a pipelined architecture to increase the system throughput, and we split the system into a live and offline processing chain. This way, real-time change detection at $3.5 \mathrm{fps}$ is achieved on images of $1920 \times 1440$ pixels. Finally, an extensive system validation featuring realistic experiments shows promising detection capabilities and robustness to, e.g., lateral displacements of up to $6 \mathrm{~m}$. @ 2019 SPIE and IS\&T [DOI: 10.1117/1.JEI.28.4.043009]

Keywords: change detection; counter-IED; computer vision.

Paper 190114 received Feb. 5, 2019; accepted for publication Jun. 10, 2019; published online Jul. 12, 2019.
\end{abstract}

\section{Introduction}

Improvised explosive devices (IED), especially roadside bombs, are a serious threat to both NATO and civilian transports in conflict areas. These homemade bombs can be fabricated by individuals using simple household items and/or chemicals, without any military background. For this reason, IED have become the preferred choice for terrorists and insurgents in conflict areas.

New and improved detection techniques are continuously investigated. An example is the ongoing research in ground penetrating radar (GPR), which uses electromagnetic induction (EMI) sensors to detect buried electronics and wires. ${ }^{1,2}$ Although these developments improve the detection capability of IED, insurgents are also continuously adapting their devices to prevent detection. One disturbing development which started in 2010 is a trend toward developing IED that cannot be detected using conventional methods. ${ }^{3}$ Although progress is made in extending GPR detection capabilities to nonmetallic conductors, ${ }^{4}$ these detection systems typically have a detection range of only a few meters. ${ }^{1}$

This changing nature of IED and the desire for early detection to facilitate a safe stopping distance require different detection strategies that are not affected by the type of applied materials and that can be added to the existing detection methods. For example, GPR can be used to verify an early detection from the new system.

Due to the strong developments in machine learning and computer vision, this technology can increasingly facilitate

*Address all correspondence to Dennis W. J. M. van de Wouw, E-mail: dennisvandewouw@gmail.com early detection. Although IED are mostly hidden from sight, practice has shown that there are often visual clues indicating the presence of an IED. We assume that the process of hiding an IED causes subtle changes to the environment, e.g., digging tracks, new dirt, rearranged branches, and or newly appeared objects that were not present during a previous visit. Another strong indicator of IED presence is the use of markers. A marker can be any (by itself innocuous) object placed on or near the road used to trigger an IED at the right time, i.e., it is detonated from a distance when the convoy reaches the marker. The markers themselves are typically not present prior to the placement of the IED, hence their presence also results in changes in the environment. For this reason, we are interested in detecting changes between the live environment as recorded while driving and the historical environment, i.e., the same environment as recorded during a previous patrol. This is also referred to as change detection.

This change detection technique is typically applied to parts of the route that are unavoidable or that pose high risk. For such areas, military forces first perform a complete clearance operation, making sure that no threats are present on the route. Directly afterward, the cleared scene can be captured by a camera vehicle. By equipping the patrolling vehicle (during a future visit) with cameras and comparing the environment with recordings from this previous visit, suspicious changes that may indicate the presence of an IED can be detected at a safe distance. This enables the convoy to take appropriate actions, such as halting for a closer inspection using different sensors, e.g., GPR, or defusal

$1017-9909 / 2019 / \$ 28.00$ ๑ 2019 SPIE and IS\&T 
of the threat. When no threats are encountered, the newly recorded video can again be used as a reference for future patrols.

Change detection from a mobile vehicle is a challenging task, where the system has to cope with illumination differences in an outdoor environment, driving trajectory differences that result in viewpoint differences between the live and historical images, but also dynamic clutter of the scene, where not all changes are suspicious. These challenges are addressed in the system design and experiments in Secs. 4 and 6, respectively.

This work describes our real-time image analysis system to be used for route-clearance operations, which is the successor of the real-time change detection system, ${ }^{5}$ which was demonstrated at the route threat detection and Clearance Technologies Challenge (NATO-SCI-256 ${ }^{6}$ ) in 2012. However, this system was based on a single camera, lacking the semantic understanding of three-dimensional (3-D) elements separated from the background. In the new work, we introduce 3-D geometry using stereo cameras, which is used to increase the robustness of the change detection system. The key of the new system is on-the-fly generation of 3-D geometry, integrated into the change detection process, to become less sensitive to parallax effects. Furthermore, the complete system should operate in real-time to facilitate a safe stopping distance of the vehicle.

In short, our contributions are

- new and complete change detection system design, incorporating 3-D geometry to increase the robustness to viewpoint changes with respect to the previous system $^{5}$ and which is no longer restricted to detecting changes on the ground surface;

- real-time design of this complex image analysis system;

- additional postprocessing filters exploiting the 3-D geometry of the scene for high reliability;

- extensive validation of this change detection system through realistic tests using our prototype vehicle.

To this end, the proposed change detection system consists of two cameras in stereo setup, a GPS with inertial measurement unit (IMU) for improved positioning accuracy and the image analysis system. These elements are meant to be installed on top of and inside a military vehicle. During driving, images of the upcoming scene are captured and stored alongside their GPS position and vehicle heading in a database. The captured images are automatically compared to historical recordings of the same environment, based on the GPS location and heading of the vehicle. All changes between the historical and live images are analyzed and only suspicious changes are reported to the operator through a graphical user interface (GUI). This process is explained in more detail in Sec. 4. Figure 1 shows the prototype vehicle used for this work.

The remainder of this paper is organized as follows. General system requirements are discussed in Sec. 2. Section 3 presents related work on change detection relevant to the context of countering IED (C-IED). Both the system hardware and software are described in detail in Sec. 4. Section 5 explains the system architecture and provides timing results for each system component and the complete

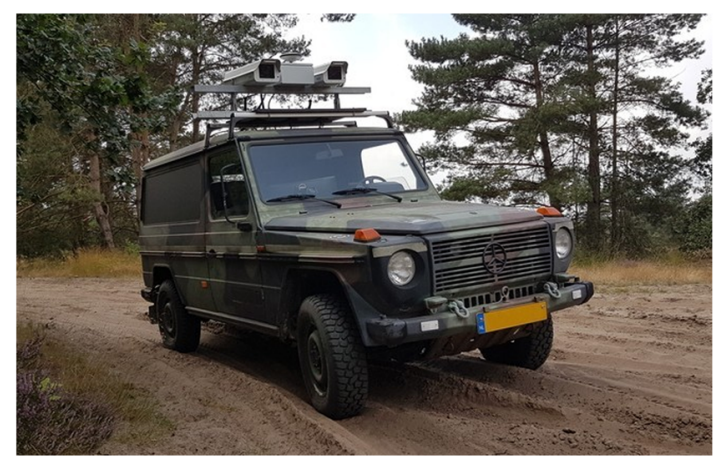

Fig. 1 System prototype including two cameras in stereo setup and a GPS/IMU for positioning.

system. Section 6 addresses the experiments and results, while Sec. 7 discusses the impact of the results within the context of C-IED. Finally, Sec. 8 concludes the paper.

\section{Requirements}

An early-warning system for route clearance poses several requirements on the driving speed and stand-off distance. In this work, we aim at a driving speed of $10 \mathrm{~km} / \mathrm{h}$ and a stand-off distance of $20 \mathrm{~m}$, i.e., the vehicle should be able to stop at 20-m distance from a threat. Furthermore, we argue that an operator typically requires $3 \mathrm{~s}$ to properly analyze a detection through the GUI. Table 1 summarizes these characteristics, where the system delay will be further addressed in Sec. 5. Figure 2 shows the corresponding distances when driving at $10 \mathrm{~km} / \mathrm{h}$. This way, a minimum detection distance of $33 \mathrm{~m}$ is obtained, i.e., a threat should be detected prior to the vehicle coming within this distance.

Table 1 System characteristics.

\begin{tabular}{lc} 
System characteristics & Value \\
\hline Minimal vehicle speed & $10 \mathrm{~km} / \mathrm{h}$ \\
Minimal stand-off distance & $20 \mathrm{~m}$ \\
Stopping distance (at $10 \mathrm{~km} / \mathrm{h})$ & $1 \mathrm{~m}$ \\
Operator decision time & $3 \mathrm{~s}$ \\
System delay (Sec. 5) & $1.5 \mathrm{~s}$ \\
\hline
\end{tabular}

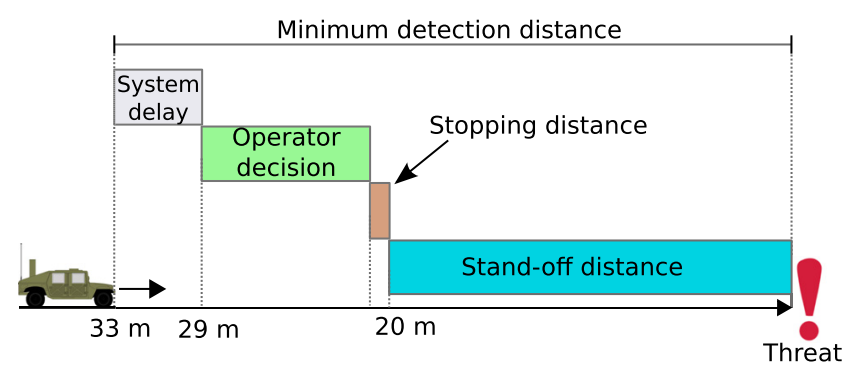

Fig. 2 Conceptual visualization of minimum detection distance. 


\section{Related Work}

Radke et al. $^{7}$ have defined change detection as follows. Given two images of the same scene acquired at different moments in time, change detection is the process of identifying the set of pixels that are significantly different between both images. In this context, unsupervised change detection is the process of automatically finding such changes without human interaction. This is explained in detail in the surveys by Radke et al. ${ }^{7}$ and Venkateswaran et al. ${ }^{8}$ Although the change detection use cases described in these surveys focus on stationary cameras and remote sensing, the commonly identified processing blocks are also applicable to change detection from a moving vehicle. The main difference lies in a more advanced image registration processing step that has to cope with strong parallax effects, due to large viewpoint differences, e.g., when driving in a different lane or in a slightly different direction.

\subsection{2-D Change Detection}

An extensive overview and ranking of various stationary change detection algorithms is given in Refs. 9 and 10 which provides a benchmarking dataset for change detection algorithms. Although several unsupervised algorithms show promising results on this dataset, these algorithms typically either use temporal information or background models that implicitly assume stationary cameras. For example, SuBSENSE by St-Charles et al. ${ }^{11}$ shows promising results on the benchmark dataset but uses temporal information to reduce sensitivity at areas with flickering (inconsistent) detections and employs downsampling to cope with small camera movements. Such models work well on (near-) stationary cameras but cannot be employed when videos are acquired from a moving platform, where the view of the scene continuously changes.

\subsection{3-D Change Detection}

More relevant to the C-IED use case is the field of 3-D change detection, since 3-D geometric information is free of illumination variations and perspective distortions. Therefore, the coregistration of 3-D data simplifies to a rigid transformation, while the registration process is unaffected by changing lighting conditions. Qin et al. ${ }^{12}$ provided an in-depth review of recent developments in 3-D change detection techniques. They argue that 3-D change detection applications are so disparate that there is no universally best method or strategy that outperforms the others. As an example, change detection techniques using LIDAR data may not work on points clouds generated using dense image matching techniques from a ground-based acquisition system.

Employing image-matching techniques from a groundbased vehicle introduces additional challenges, such as uncertainties in the 3-D data and irregular sampling. Both are especially relevant for stereo matching, where the estimated depth may be noisy and where matching errors may occur, e.g., on texture-less areas. Moreover, the perspective of the images acquired from a ground-based vehicle will cause the number of 3-D points to decrease with increasing distance to the vehicle, resulting in a nonuniform distribution of the point cloud.

Direct comparison of such point clouds usually produces many artifacts. Therefore, the 3-D space is typically converted to a digital elevation map (DEM) for aerial change detection, ${ }^{13}$ where changes in either the height or the volume of the model denote a change. The 3-D space can also be divided into voxels for ground-based change detection, where voxels with significant color differences denote changes. However, due to the nature of ground-based imagery, a DEM is not suited for detecting our small changes of interest in an environment that is not entirely captured by the surface model, e.g., a change at the side of a tree is not captured by the DEM. Voxels can capture the entire scene, although voxalization results in a high computational burden, especially when small changes of up to $10 \times 10 \times 10 \mathrm{~cm}$ need to be detected. Increasing the granularity of the voxels to reduce their amount reduces the ability to detect small objects. For this reason, such methods have been limited to small scenes, large objects, and low-resolution voxels. Bláha et al. ${ }^{14}$ proposed to use a coarse-to-fine strategy to form adaptive voxels, thereby reducing the memory and computational footprint. This hierarchical approach refines the reconstruction only in regions that are likely to contain a surface. Although this works well on remotely sensed and aerial data, it may omit the small and thin structures that are of interest in our C-IED scenario.

Xiao et al. ${ }^{15}$ combined occupancy grids and a distancebased method for 3-D change detection in mobile laser scanning data, where they avoid voxalization altogether. The occupancy grid is constructed using ray tracing and is employed to distinguish occlusions and dynamic objects from real changes. Change detection is then performed in 3-D by analyzing the point-to-triangle distance, which makes it robust to density variations in the point cloud. Although this approach works well for structural change detection in urban environments, our objects of interest are too small to be detected using this approach.

Taneja et al. ${ }^{16}$ also proposed a change detection technique for detecting changes in the geometry of a city, where they use panoramic images captured from a driving car. Registration is performed by segmenting the scene and aligning building outlines in the images with respect to a cadastral 3-D model. Furthermore, they check for inconsistencies in the geometry by evaluating the color consistency. For this purpose, they project the image texture onto the 3-D model and use it to synthesize an image from a previous viewpoint. This image is then subtracted from the panoramic image taken at that location to find the differences. To correct for subsampling (blurring) artifacts that occur during viewpoint synthetization, they simulate the same blurring artifacts on the original image prior to comparison. A color difference then represents an error in the geometry that may be corrected. Change detection is performed per voxel, with a resolution of $1 \mathrm{~m}^{3}$. Increasing the voxel resolution to the size of our objects of interest would lead to unreasonable execution times. Moreover, the initial registration requires a cadastral 3-D model, which is not available in the context of C-IED. Zhou et al. ${ }^{17}$ used a similar technique where they construct DEMs from aerial LIDAR data, generate image projections, and look for color difference that denotes inconsistencies in the model geometry.

\subsection{5-D Change Detection}

Despite the presented advantages of 3-D change detection, we argue that purely 3-D change detection is not suited for 
the C-IED scenario, where changes may be small and may not even cause geometrical changes, e.g., digging tracks may not necessarily cause volumetric changes. We therefore expand our overview toward combined two-dimensional (2-D) and 3-D change detection, where 3-D geometry is employed to improve scene alignment and change detection is typically performed in 2-D.

Haberdar and Shah ${ }^{18}$ performed change detection from a moving vehicle, where they assume that relevant changes occur on the dominant plane in the scene, i.e., the ground plane, when recording from a vehicle. First, they apply a layer-based segmentation on both the texture and disparity estimates to find the dominant plane in each image. Next, the dominant planes are aligned using a homography transformation, after which change detection (on the ground plane) is performed in 2-D. Change detection is performed by dividing the image into square subregions, after which each region is evaluated for the integrated texture- and intensity gradient features. It should be noted that in the case of viewpoint changes, this method does not properly register those parts of the scene that do not belong to the ground plane.

Morales et al. ${ }^{19}$ do not limit their registration to the ground surface. Instead, they use piecewise linear transformations to avoid parallax issues when registering the entire scene. First, they generate a triangular mesh of the scene using the Delaunay algorithm. Each triangle is then transformed separately using a linear transform, based on feature correspondences within that triangle. Once registration is completed, principal component analysis is used to find changes between the aligned historic and live image, where the analysis is limited to the brightness of grayscale images. However, the approach does not operate in real time on our target resolution of $1920 \times 1440$ pixels. Moreover, the piecewise local geometric model for each segment relies on accurate point-to-point correspondences within that segment. In our experience, it is not always possible to obtain accurate point correspondences in every part of the scene, especially when texture-less road surfaces are involved.

Van de Wouw et al. ${ }^{20,21}$ proposed a 2.5-D registration approach to avoid parallax effects, when aligning images with typical driving-related viewpoint differences. For this purpose, they build a simplified 3-D model of the historical scene, which is transformed to the 3-D coordinate frame of the live camera. Projecting this model back to 2-D results in a regular image of the historical scene, as if it was captured from the live camera viewpoint. This strongly resembles the approach of Taneja et al., ${ }^{16}$ where viewpoint synthetization is employed to distinguish between real geometry changes and those caused by dynamic changes. Next, change detection is performed by taking the maximum absolute difference over the color channels (RGB) and thresholding the result, followed by postprocessing techniques to reduce the false alarm rate.

Alcantarilla et al. ${ }^{22}$ proposed a change detection approach for finding changes from street-level images between different seasons. For this purpose, GPS and odometry are used to estimate a depth map of the scene and create a dense 3-D reconstruction of the scene. Images are then coarsely aligned using multisensor SLAM, after which a deconvolutional network performs pixel-based change detection. However, the objects of interest in these images are significantly larger than our objects of interest and the approach requires the availability of training data. Moreover, the execution time of $2 \mathrm{~s}$ per frame does not allow for real-time operation.

In this paper, we adopt the 2.5-D registration approach of Van de Wouw et al., ${ }^{21}$ although we use a different approach for the change detection and false alarm reduction. We achieve real-time performance of the complete dualcamera change detection system, by introducing a pipelined design and executing the most time-consuming processing tasks on graphical processing units (GPUs). Moreover, change detection is split into a live and off-line processing chain, where the latter preprocesses the historical images to reduce computational load during live processing. Extensive validation of the system is shown in Sec. 6, where we demonstrate the robustness to common challenges in operational scenarios.

\section{Proposed System}

The system captures stereo images while driving and computes a disparity map for each image pair. Both the images and depth map are stored in a database, to be used as historical data during future patrols. Next, the historical reference frame that best resembles the live viewpoint is retrieved from the database, using the GPS position and heading of the vehicle. This historical frame is aligned to the live camera by synthesizing the historical scene from the live camera viewpoint, e.g., we obtain a 2-D image of the historical scene as if viewed from the live camera. This synthesized historical image is compared to the live image, where all changes are analyzed by a change-analysis algorithm. Finally, all suspicious changes are shown to the user through an interactive GUI.

We split the complete change detection system into two separate processing chains, as shown in Fig. 3. The on-lineprocessing chain involves all processing tasks related to live change detection during a patrol, e.g., comparing the images and analyzing the changes. The second, off-line preprocessing chain prepares all recorded images to be used as (historical) reference data in future patrols and is typically performed overnight. This division into an on-line and off-line chain enables the system to use more expensive preprocessing algorithms, which would otherwise slow down and delay the live change detection. The precomputed (depth) data can then be directly loaded from the database during live processing.

Each processing block from Fig. 3 is discussed in detail below.

\subsection{Video Capturing}

Images are captured by two cameras in a stereo setup and are stored as 5-MegaPixel JPEG2000 encoded images $(2560 \times 1920$ pixels $)$, where encoding takes place on the FPGA inside the camera. To limit the amount of data to be processed and stored, images are not recorded as a video stream. Instead, a single stereo image is captured every $0.5 \mathrm{~m}$. This is achieved by sending an external trigger over a CAN interface, whenever the GPS/IMU indicates sufficient distance was traversed. This mechanism also allows to assign an accurate GPS position and the viewing direction of the camera to each recorded image. These metadata are stored to the database alongside the image data. This is also visualized in Fig. 4. For efficiency reasons, the images 

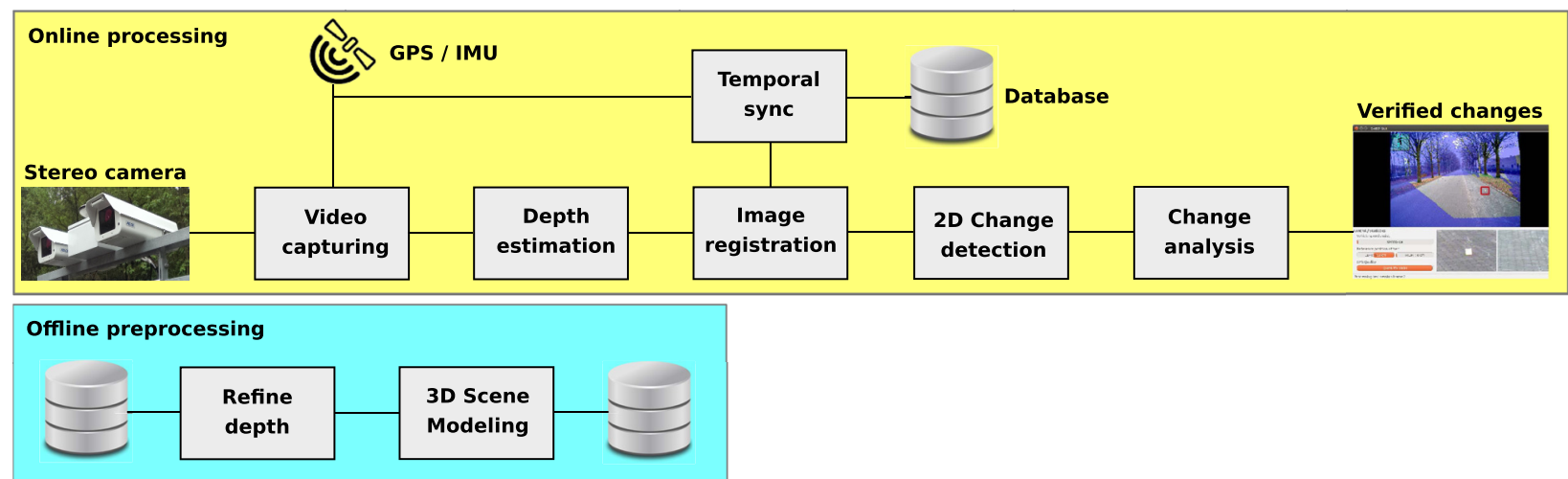

Fig. 3 Proposed data flow for both on-line-change detection and off-line preprocessing.

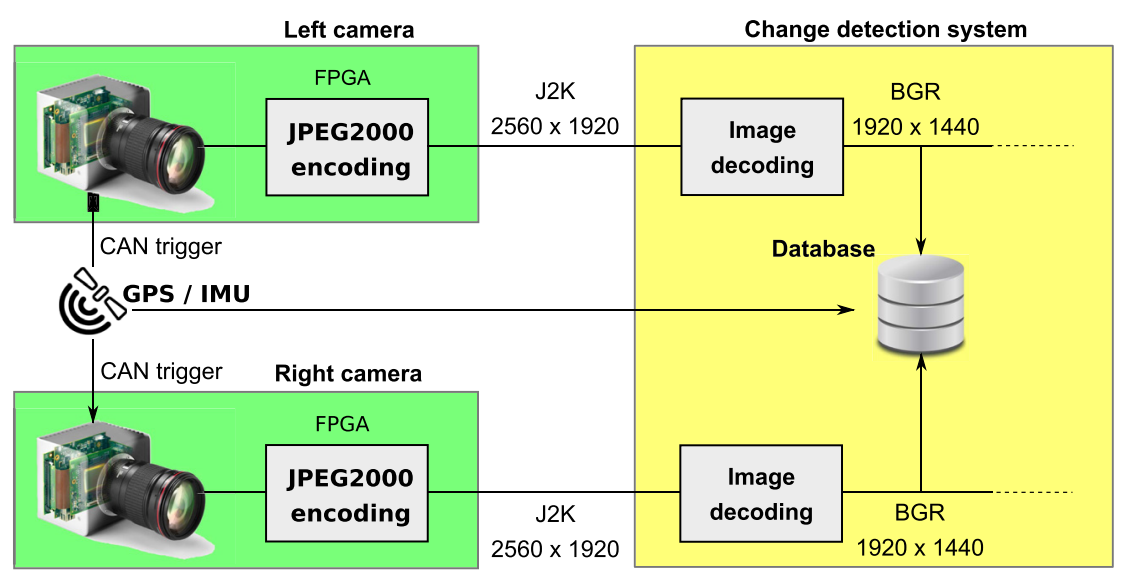

Fig. 4 Schematic overview of the video capturing.

themselves are stored directly on disk, while the database contains only references to the data. This also helps to keep the database size small and manageable.

Throughout this work, all images are decoded to a resolution of $1920 \times 1440$ pixels. This resolution constraint is currently imposed by the disparity generation, discussed in the next section. Since the computing time of the disparity map rises more than quadratically with increasing resolution, a higher resolution cannot be processed in real time using the current hardware system.

\subsection{Depth Estimation}

The dual-camera setup enables stereo-processing to obtain a disparity map. For the computation of this disparity map, we adopt (and modify) the work of Ranft and Strauß. ${ }^{23}$ They argued that window-based local methods, such as block matchers, can be implemented very efficiently, but are prone to errors. Especially block matching on nonfrontal surfaces is complicated, since the distance to the camera is not constant across the matching window. This is typically the case for the road surface, which is not parallel but slanted with respect to the image plane when recorded from a vehicle. Ranft and Strauß compensated for the slanted (ground) surface by applying a shearing transform to the right stereo image. Next, they applied a modified Census transform to mitigate illumination differences, by assigning each pixel a 16-bit descriptor based on comparisons with eight neighboring pixels. The Hamming distance is then used as a metric for the similarity between the Census maps of the left and right images.

Where Ranft and Strauß search for local maxima in the histograms of disparity gradients to find the required shearing gradient $g=\partial d / \partial v,^{23}$ this approach was found to be noisy on our high-resolution stereo images. Instead, we employ a plane-fitting algorithm directly on the originally obtained disparity values, after which the shearing parameters are found through a RANSAC scheme that solves for $d=a+b u+c v$. Here, $u$ and $v$ represent screen coordinates, and $b$ and $c$ denote the horizontal and vertical disparity gradient, respectively. In this case, we set $g=c$.

A total of two disparity maps is computed for each stereo pair. One disparity map without shearing (frontal) and one with shearing. The latter is merged with the frontal-disparity map using a winner-takes-all voting scheme, resulting in a single disparity map that has good disparity estimates on both frontal objects and the ground plane.

To further improve the quality of the disparity map, we apply bidirectional matching for both the frontal and sheared disparity estimation. A straightforward bidirectional matching would result in twice the computational complexity, which is not feasible within our real-time system. Therefore, we apply the backward check of the bidirectional matching on $4 \times$ downsampled versions of the images. Experiments show that this significantly reduces noise in the disparity map, while only increasing the computation time by $12 \mathrm{~ms}$ to a total of $132 \mathrm{~ms}$ for images of $1920 \times 1440$ pixels. 


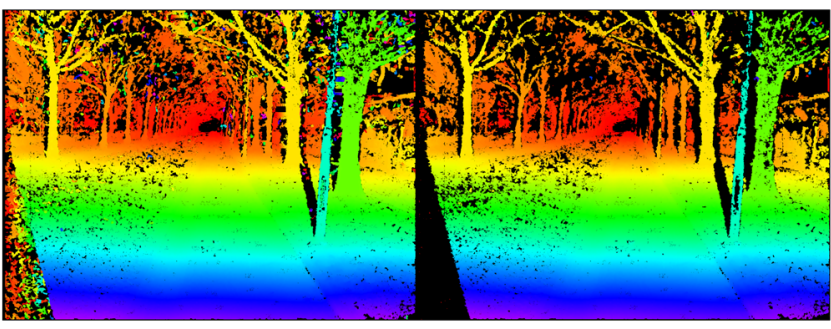

Fig. 5 (a) Original disparity map and (b) the improved disparity map after the downsampled bidirectional check. The noisy estimates in nonmatchable areas have been removed, where such areas are now shown as consistent black (invalid) areas. The blue color stands for nearby distances and the red color indicates larger distance to the vehicle.

Figure 5 shows the effect of our efficient bidirectional check, which effectively removes noise in occluded areas of the disparity map.

Finally, a straightforward speckle filtering and median blurring is applied to further refine the disparity map. The speckle filtering removes disparity blobs smaller than 100 pixels that deviate more than two full disparity values from their surroundings. For the median blurring, a support window of 3 pixels is used to remove small holes in the disparity map. The full approach is summarized in Fig. 6.

\subsubsection{Compensation for lens vibration}

It was observed during our experiments that the internal components of the camera lenses reacted to the engine and driving-related vibrations of the car. As a result, both the left and right principal points shifted independently from each other, causing the stereo calibration to become invalid. In fact, corresponding points were no longer located on the same scan line in the rectified images, thereby making disparity estimation inaccurate or even impossible. To compensate for this vibration, we employ feature matching to find the median value of the vertical motion between the rectified images. By shifting the right-rectified image by this value, the images become rectified again. This is conceptually visualized in Fig. 7, where corresponding points once more lie on the same scanline after the vertical shift. It should be noted that only vertical vibration can be compensated this way, as horizontal vibrations cannot be distinguished from a different disparity estimate. However, additional experiments have revealed that, in our case, the depth measurements were not affected and the vibrations were apparently dominantly vertically oriented.

\subsection{Temporal Synchronization}

In order to compare the live and historical scene, the system needs to find the historical image that best resembles the live scene. Instead of simply loading all images in the neighborhood and computing the visual overlap, the viewpoint overlap is estimated using the meta data only. This selection procedure is based on the GPS position and heading of the vehicle corresponding to the live and historical image, where the historical image with the largest top-down viewpoint overlap is selected (Fig. 8).

More specifically, this approach finds the historical image that minimizes the parametric cost function $C_{o}$

$C_{o}=-a \mathrm{FOV}_{\text {overlap }}+b \Delta x+c \Delta y$,

where $\mathrm{FOV}_{\text {overlap }}$ denotes the top-down viewpoint overlap denoted in blue in Fig. 8, $\Delta x$ represents the lateral displacement and $\Delta y$ portrays the forward displacement of the vehicle.

Equation (1) is basically a trade-off between the effective operational range of change detection versus the accuracy of detected changes. Change detection can only be employed within the overlapping field-of-view (FOV), while its accuracy degrades with larger displacements. Taking into account that the $\mathrm{FOV}_{\text {overlap }}$ has a maximum value of unity, $\Delta y$ is at most half the sampling distance $(0.25 \mathrm{~m})$ and $\Delta x$ can go up to $7 \mathrm{~m}$, the latter two should receive a lower weight. Moreover,

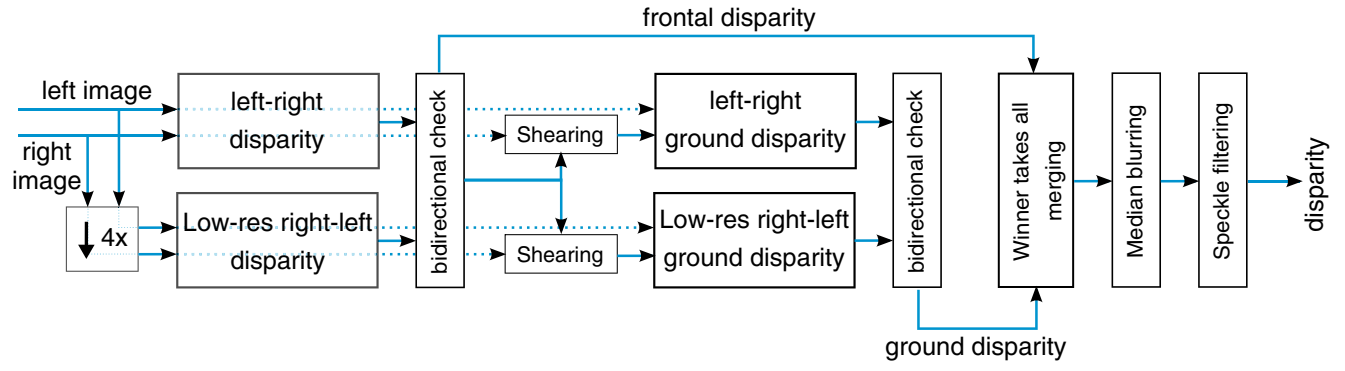

Fig. 6 Schematic overview of the disparity generation.

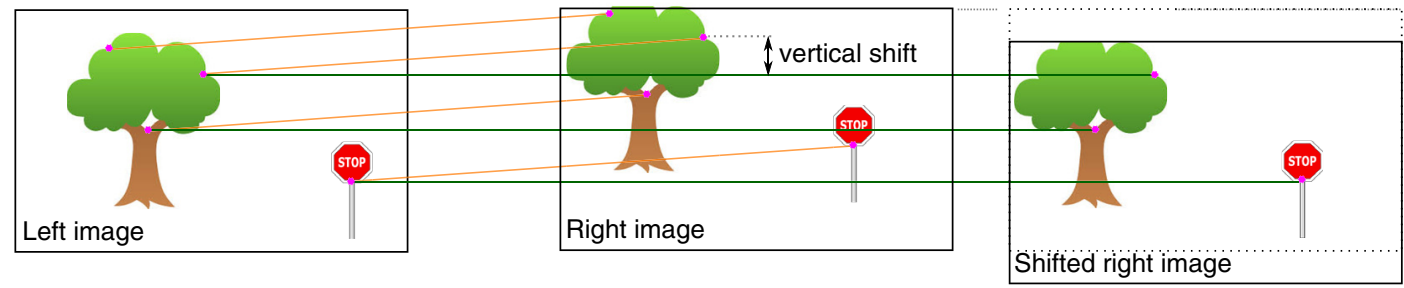

Fig. 7 Conceptual impression of compensation for lens vibration. The orange and green lines denote corresponding points in the rectified images prior to and after the vertical shift, respectively. 

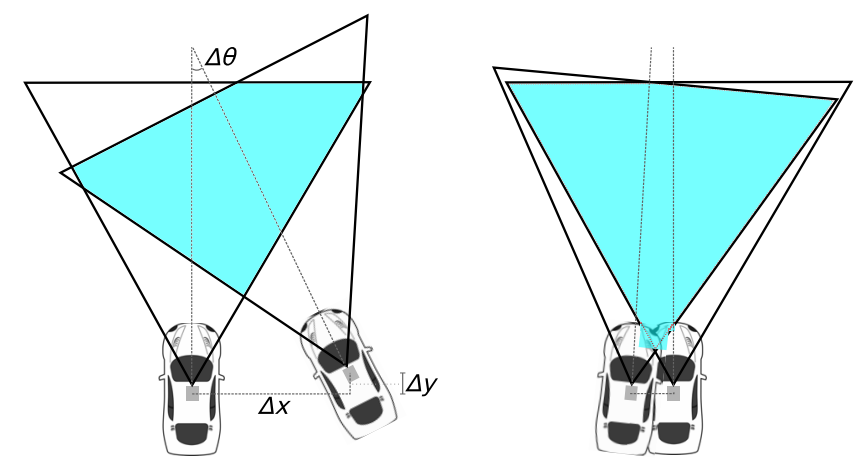

Fig. 8 Top-down viewpoint overlap between the live and historical viewpoint used in the temporal synchronization. The example on the right shows a better viewpoint overlap with smaller angular difference $\Delta \theta$, lateral displacement $\Delta x$, and forward displacement $\Delta y$.

since the system is more sensitive to horizontal than to vertical displacements, the parameter values have been empirically set to $a=0.7, b=0.2$, and $c=0.1$, to compensate for this imbalance.

Once the best resembling historical image is known, the system only loads that specific image and its corresponding depth data.

\subsection{Image Registration}

For the image registration, we adopt the 2.5-D hierarchical alignment as introduced by Wouw et al. ${ }^{20}$ This approach synthesizes the historical scene as if viewed from the live camera. First, the 3-D transformation between the live and historical view is estimated, using an existing feature-based method. ${ }^{24}$ This approach uses synthesized views, similar to the shearing transform in the disparity estimation, to improve scene similarity. This enables to find more accurate feature correspondences, which in combination with the depth map are used to find the 3-D transformation (pose difference) between the live and historical camera. Next, the textured 3-D model of the historical scene (Sec. 4.7) is transformed according to the estimated pose difference, effectively transforming it to the coordinate system of the live camera. The transformed model is then projected back to 2-D, yielding an image of the historical scene from the live camera viewpoint. This initial registration is further refined using local template matching. ${ }^{20}$ Figure 9 shows a live image and the corresponding registered historical reference image.

Whereas the original method was restricted to the ground surface, this work employs the extended scene modeling described in recent work, ${ }^{21}$ which enables the system to render the entire historical scene. This is briefly described in Sec. 4.7.

\subsection{2-D Change Detection}

In the original change detection systems, ${ }^{5,20}$ RGB differencing in combination with adaptive thresholding is employed to obtain a binary change mask, where the adaptive thresholding is based on a global threshold. However, differencing on the RGB images directly in combination with a global threshold was found to be too sensitive to local illumination changes, such as shadows.

In this work, we acknowledge that illumination differences between the live and historical reference scene will occur and that a global threshold either loses relevant changes or allows large shadows and bright areas to result in false changes. By focusing on local area statistics instead, small changes are favored, whereas large shadows and overexposed areas are suppressed.

To better cope with illumination differences, we transform the images to the CIE $L * a * b *$ color space, which was shown to properly separate the luminance (L-channel) from the color (AB-channels). ${ }^{25}$ From the color channels, specific color differences are then computed by taking the absolute difference between the live and historical individual ABchannels and from those differences the maximum value for each pixel. Next, local adaptive thresholding is applied to obtain a color-change mask. As our changes of interest may not necessarily feature color differences, the same processing is also applied to the luminance channel, where a different threshold is applied to smoothly handle minor illumination changes.

Both the color- and luminance-change masks are refined using morphological filtering for removing noisy changes. First, a $7 \times 7$ closing filter is employed to merge multiple (partial) detections on the same object. Second, a $3 \times 3$ opening filter is employed to remove all changes that are smaller than 9 pixels. Such small changes are too small to distinguish from noise. Next, clustering by means of a connected components algorithm results in a list of color and luminance changes, where each change is represented by a bounding box. Finally, the list of color and luminance changes is merged to a single list of changes to be further analyzed by the change analysis. The entire procedure is summarized in Algorithm 1.

\subsection{Change Analysis}

The list of changes from the 2-D change detection may contain irrelevant changes, such as leafs moved by the wind, small registration errors, and or strong shadows. To

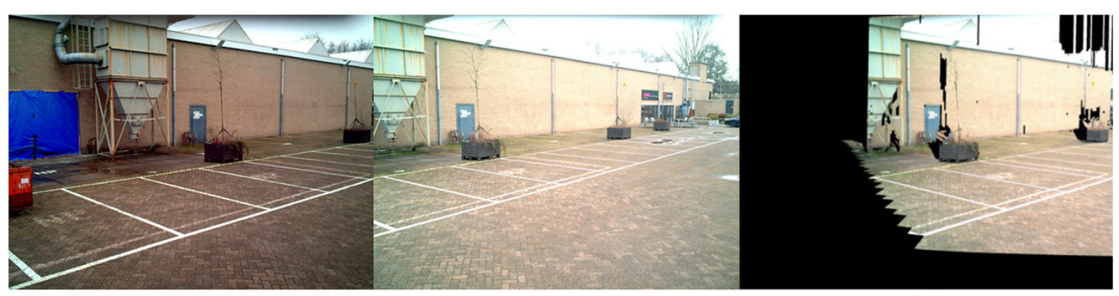

(a)

(b)

(c)

Fig. 9 (a) Live image and (b) historical reference image and (c) the historical image registered to the live image. 
Algorithm 1 2-D change detection.

Input: $I^{L}$, live image, $I^{H}$ historical image aligned to live

Output: $C$, list of changes

Variables: $d$, absolute image difference; $\sigma$, local standard deviation of absolute differences; $M$, preliminary binary change mask; $\alpha$, threshold for luminance; $\beta$, threshold for color.

1: procedure DETECTCHANGES $\left(I^{L}, I^{H}\right)$

2: $\left[L^{L}, A^{L}, B^{L}\right]=$ convertToCieLAB $\left(I^{L}\right)$;

3: $\left[L^{H}, A^{H}, B^{H}\right]=$ convertToCieLAB $\left(I^{H}\right)$;

4: $\quad$ for each pixel $p_{i, j}$ do

5: $\quad d_{i, j}^{\text {lumi }}=\left|L_{i, j}^{L}-L_{i, j}^{H}\right|$

6: $\quad d_{i, j}^{\text {color }}=\max \left(\left|A_{i, j}^{L}-A_{i, j}^{H}\right|,\left|B_{i, j}^{L}-B_{i, j}^{H}\right|\right)$

7: end for

8: $\quad$ for each pixel $p_{i, j}$ do

9: $\quad \sigma_{i, j}^{\text {lumi }}=$ ComputeStdevInRegionAroundPixel $\left(d^{\text {lumi }}, i, j\right)$

10: $\quad \sigma_{i, j}^{\text {color }}=$ ComputeStdevInRegionAroundPixel $\left(d^{\text {color }}, i, j\right)$

11: $\quad M_{i, j}^{\text {lumi }}=\left\{\begin{array}{cc}255, & \text { if }\left(d_{i, j}^{\text {lumi }}>\alpha * \sigma_{i, j}^{\text {lumi }}\right) \\ 0, & \text { otherwise }\end{array}\right.$

12: $\quad M_{i, j}^{\text {color }}=\left\{\begin{array}{cc}255, & \text { if }\left(d_{i, j}^{\text {color }}>\beta * \sigma_{i, j}^{\text {color }}\right) \\ 0, & \text { otherwise }\end{array}\right.$

13: end for

14: $\quad M^{\text {lumi }}=$ morphologicalFiltering $\left(M^{\text {lumi }}\right)$

15: $\quad M^{\text {color }}=$ morphologicalFiltering $\left(M^{\text {color }}\right)$

16: $\quad C=$ connectedComponents $\left(M^{\text {lumi }}\right)+$ connectedComponents $\left(M^{\text {color }}\right)$

17: $\quad$ return $C$

18: end procedure

selectively report changes to the operator, several postprocessing techniques are applied, which are listed as follows.

- 2-D size:

This filter reduces false detections due to sensor noise, where changes with an area below 30 pixels are rejected. Considering that an object of $9 \times 18 \mathrm{~cm}$ at a distance of $33 \mathrm{~m}$ already covers 44 pixels, this is a valid assumption for significantly reducing false alarms.

- Real-world size:

The frontal area of each change blob is estimated in real-world coordinates as

$S_{r w}=\left(X_{\max }-X_{\min }\right) *\left(Y_{\max }-Y_{\min }\right)$.

Typically, changes below $50 \mathrm{~cm}^{2}$ are rejected, although this threshold can be adjusted for different terrains. It should be noted that the stereo camera is mounted under a forward-downlooking angle (pitch), which ensures that flat objects, e.g., digging tracks, also yield a difference in height $(d Y)$ and thus a frontal surface.

- 3-D region of interest:

Change reporting is restricted to the operational range of the stereo camera, where the depth measurements are still sufficiently accurate to allow for the aforementioned filter. Changes that are farther away will eventually become in range.

- Dissimilarity:

The sum of absolute pixel differences between the mean-normalized live and the mean-normalized historical RGB image on and around a change is used as a dissimilarity measure. Here, we sum over the maximum of the absolute difference among the R, G, and $\mathrm{B}$ color channels. This dissimilarity is computed as

$$
D=\frac{1}{N} \sum_{l, h \in C} \max _{r, g, b}\left(\left[\begin{array}{c}
\left|\left(l_{r}-\bar{l}_{r}\right)-\left(h_{r}-\bar{h}_{r}\right)\right| \\
\left|\left(l_{g}-\bar{l}_{g}\right)-\left(h_{g}-\bar{h}_{g}\right)\right| \\
\left|\left(l_{b}-\bar{l}_{b}\right)-\left(h_{b}-\bar{h}_{b}\right)\right|
\end{array}\right]\right),
$$

where $C$ denotes the set of all corresponding $l$ live and $h$ historical pixel values within the change region, $\bar{l}$ and $\bar{h}$ denote their averages, and $N$ denotes the number of pixels in that change. A change is rejected if the dissimilarity $D$ is below a threshold $\tau=70$.

- Temporal consistency:

To reduce the amount of sporadic false detections, as well as prevent detections on dynamic objects, e.g., moving traffic participants, temporal filtering is applied. Here, changes are only accepted when they appear in at least two successive frames at the same 3-D location.

Any remaining changes are presented to the operator through the GUI, presented in Sec. 4.8.

\subsection{3-D Modeling}

This module first improves the depth estimation from Sec. 4.2 and then constructs a simplified 3-D model to be used in the scene registration (Sec. 4.4). First, a bilateral disparity filter ${ }^{26}$ is applied to enhance the shape accuracy of the disparity map. Next, a textured 3-D model is constructed. Instead of computing a full model of the 3-D world, we employ a simplified box-diorama model. ${ }^{21}$ This model consists of a 3-D triangular mesh of the nonlinear ground surface, where the objects in the scene are superimposed as rectangles, perpendicular to the ground surface. After texture has been projected onto this mesh, it can be transformed to a (future) live camera pose and projected back to a 2-D image, resulting in a registered image [Fig. 9(c)].

\subsection{Graphical User Interface}

The GUI of the prototype system is shown in Fig. 10. This interface features a touch screen mounted inside the vehicle, where detected changes are presented to the operator by a bounding box on top of the live view. Next, the operator can touch a change or any other part of the screen, to obtain a zoomed view of that specific part of the live image, as well 


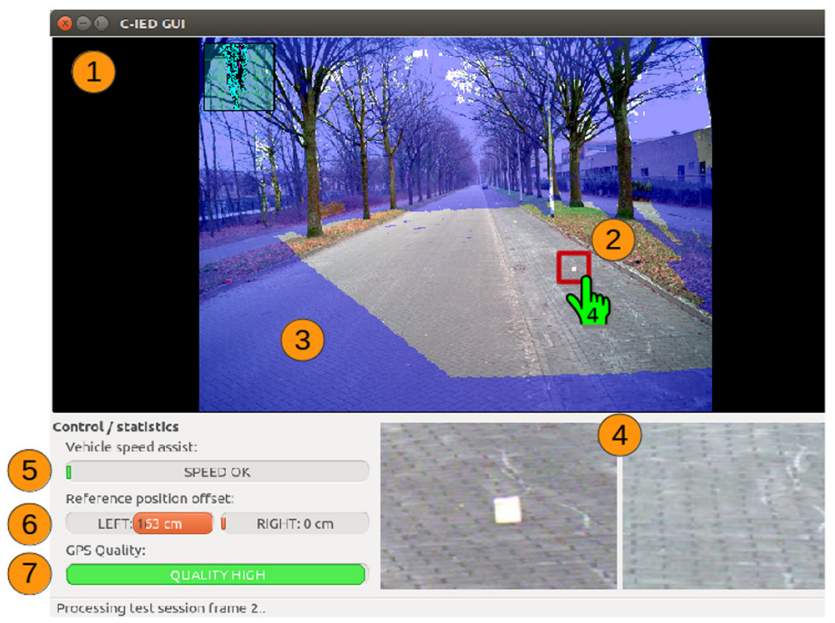

Fig. $10 \mathrm{GUI}$ of the prototype change detection system, featuring: (1) live view; (2) detected change denoted by a bounding box; (3) blue indicates the image area outside the operational range, e.g., outside the viewpoint overlap between the live and historical images or due to occlusions; (4) zoomed live and historical view of the area around the selected detection; (5) speed indicator; (6) lateral displacement of the vehicle with respect to the historical drive; (7) GPS quality.

as a zoom of the corresponding area in the historical view [Fig. 10(4)]. This allows for a quick comparison and enables the operator to make an informed decision whether to stop the vehicle or ignore the alarm. Furthermore, the system advises on the current driving speed and trajectory, by indicating that the speed may be too high (the sample interval becomes too large) and visualizing the trajectory displacement of the vehicle with respect to a previous drive. The operator may then decide to alter the current driving speed and/or trajectory to improve the system accuracy.

\section{Real-Time Design}

The prototype system consists of a hexa-core CPU (Core i7 3960X from 2011) and two high-end GPUs (TitanX with Maxwell architecture). This work features three design choices that facilitate real-time operation of the change detection system. First, to increase the system throughput, the change detection system is subdivided into five stages, which are executed in parallel through a pipelining approach. Second, the most time-consuming processing tasks are executed on GPUs. Third, all processing that should be applied only to the historical data was removed from the live processing chain. For this purpose, the change detection processing has been split into a separate live (on-line) and off-line (pipelined) processing chain. Each of these design choices is now discussed individually.

\subsection{Pipelined Architecture}

To fully exploit the six cores of the CPU and the two GPUs, the set of algorithms is split up into five parts (stages), where each stage is executed in parallel on different CPU cores and/or GPUs. This technique is also referred to as pipelining. In this work, the number of pipeline tokens is equal to the number of stages, meaning that we execute five stages in parallel. In the ideal situation, where each stage takes the same amount of processing time, the pipelining increases the throughput of this example with a factor of 5. However, in reality, the computing resources are limited, the processing
Table 2 Change detection algorithm timing with and without pipelining and the distribution over the different pipeline stages. The fourth column presents timings acquired during pipelined operation under full system load, i.e., when all five stages execute simultaneously. The overhead typically involves small processing tasks left out of the table and waiting for resources to become available.

\begin{tabular}{|c|c|c|c|c|}
\hline Stage & Processing task & $\begin{array}{l}\text { Sequential } \\
\text { time (ms) }\end{array}$ & $\begin{array}{l}\text { Pipelined } \\
\text { time (ms) }\end{array}$ & $\begin{array}{l}\text { CPU/ } \\
\text { GPU }\end{array}$ \\
\hline \multirow[t]{6}{*}{1} & Image decoding & 44 & 50 & GPU \\
\hline & Database queries $^{\mathrm{a}}$ & (2) & (2) & CPU \\
\hline & $\begin{array}{l}\text { Rectification and compensate } \\
\text { lens vibration }\end{array}$ & 29 & 37 & Both \\
\hline & Disparity estimation (live) & 132 & 153 & GPU \\
\hline & Overhead & 8 & 14 & \\
\hline & & 213 & 254 & \\
\hline \multirow[t]{5}{*}{2} & Disparity map refinement & 32 & 40 & CPU \\
\hline & Point cloud generation & 20 & 26 & CPU \\
\hline & Ground surface mesh & 151 & 220 & CPU \\
\hline & Overhead & 1 & 1 & \\
\hline & & 204 & 287 & \\
\hline \multirow[t]{8}{*}{3} & Temporal synchronization & 0 & 0 & CPU \\
\hline & Loading depth data & 2 & 2 & CPU \\
\hline & $\begin{array}{l}\text { Find feature correspondences } \\
\text { and estimate 3-D } \\
\text { transformation }\end{array}$ & 90 & 155 & Both \\
\hline & Image decoding & 42 & 77 & GPU \\
\hline & $\begin{array}{l}\text { Rectification and compensate } \\
\text { lens vibration }\end{array}$ & 11 & 16 & Both \\
\hline & Ground and obstacle masks & 12 & 15 & CPU \\
\hline & Overhead & 0 & 0 & \\
\hline & & 157 & 265 & \\
\hline \multirow[t]{5}{*}{4} & Simulate live viewpoint & 35 & 46 & GPU \\
\hline & Registration refinement & 135 & 135 & GPU \\
\hline & Apply warping to disparity & 64 & 72 & GPU \\
\hline & Overhead & 9 & 26 & \\
\hline & & 243 & 279 & \\
\hline \multirow[t]{3}{*}{5} & $\begin{array}{l}\text { 2-D change detection and } \\
\text { change analysis }\end{array}$ & 210 & 221 & CPU \\
\hline & Interactive GUI & 13 & 13 & CPU \\
\hline & & 223 & 234 & \\
\hline
\end{tabular}

aPerformed in parallel; see Fig. 11. 


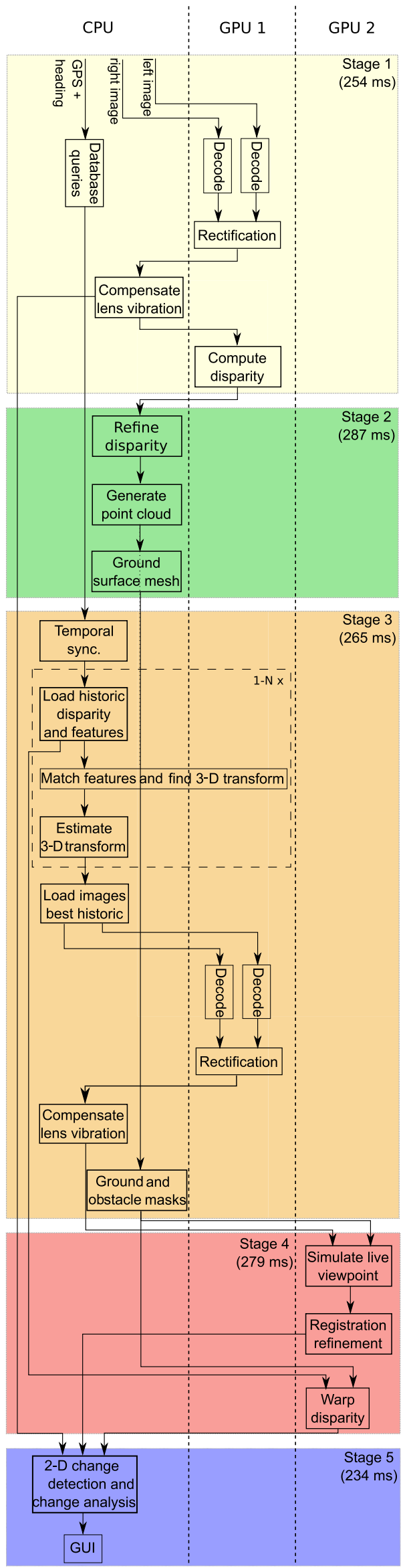

Fig. 11 Chronological processing layout. time in each stage is not necessarily equal and the pipeline stages may influence each other, as shown in Table 2, which indicates the sequential execution times, the division of the live-processing tasks into the five pipeline stages and their corresponding delays. This table also exhibits if the specific task is executed on the CPU or on a GPU.

The processing tasks from Table 2 are also shown in Fig. 11, which portrays the chronological processing layout. In this figure, arrows denote causal dependencies and arrows crossing the vertical dotted line represent CPU to GPU transfers and vice versa. This figure shows an additional loop in the processing design of stage 3 , which is outside the scope of this paper. This block enables the system to find a 3-D transformation between the live and multiple historical views and can be used as a fall-back algorithm in case of low GPS accuracy, i.e., when the method described in Sec. 4.3 cannot be employed. However, during our extensive testing period covering more than 30 recordings under different recording conditions, acquired over different days in both rural and forest environments, GPS accuracy was always found to be sufficiently accurate to employ the temporal synchronization algorithm from Sec. 4.3. Nevertheless, this repetitive design is the reason why the historic images are loaded separately from the historic depth data and features. This way, the system only needs to decode a single historical image pair, avoiding expensive image decoding of all historical pairs.

The reader may wonder why the computation of the ground and obstacle masks on the CPU in stage 3 (Fig. 11) are not performed in parallel with the image decoding on the GPU. Although the system currently employs GPU-decoded JPEG2000 images, it is expected that future versions of the system may feature different image formats, requiring CPU usage instead. For this reason, and because the mask generation is not that expensive, it was decided to execute these two processing tasks sequentially.

Figure 12 shows the achieved timing distribution over the different stages. In the ideal situation, this distribution should be evenly distributed, since the stage with the longest processing time is the bottleneck for the system throughput. Therefore, it is sometimes better to shift certain processing tasks to a different stage, even though they conceptually belong together, e.g., the disparity and disparity refinement (see Table 2). With the current division, a throughput of $3.5 \mathrm{fps}$ is achieved versus $1 \mathrm{fps}$ in sequential mode. By this result, we motivate that a frame rate of $3.5 \mathrm{~Hz}$ gives sufficient detection opportunities during the 3-s decision interval of the operator (Fig. 2), yielding at least 10 detection moments.

The timings shown in Table 2 are measured under full system load, i.e., when all five stages execute in parallel.

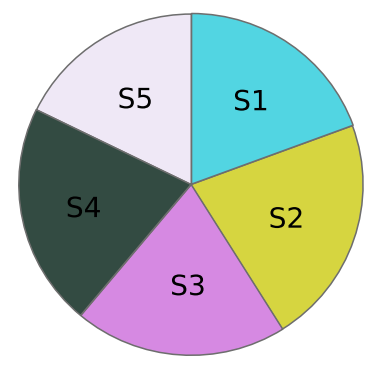

Fig. 12 Achieved timing distribution over the pipeline stages, which is approximately evenly distributed. 
As an example, the disparity estimation in sequential mode takes 132 ms. However, when parallel stages claim the same GPU, the processing time increases to $153 \mathrm{~ms}$ due to the limited availability of resources. The same holds for the CPU, where the ground surface mesh takes only $151 \mathrm{~ms}$ when executed separately versus $220 \mathrm{~ms}$ during pipelined execution. Making additional resources become available, i.e., a newer CPU with more cores, will significantly improve the execution times of all CPU tasks in Table 2. The same holds for the GPU, where preliminary experiments show a speedup of $30 \%$ when switching from the TitanX with Cuda6 (one of the third-party libraries is currently limited to CUDA 6) to the 1080Ti with CUDA8.

If the number of pipeline tokens equals the number of pipeline stages, as is the case in our system, the total system delay is determined by the slowest pipeline stage as follows:

$\tau_{\text {total }}=(M-1) \cdot \max \left(\tau_{1}, \ldots, \tau_{M}\right)+\tau_{M}$,

where $M$ represents the number of pipeline stages and $\tau_{1} \ldots \tau_{M}$ denote the delay of each stage. The output of the last stage is immediately available after processing and does not need to wait for the next pipeline stage, hence the separate $\tau_{M}$ in the formula. This results in a processing delay of $1.38 \mathrm{~s}$ when pipelining is applied. Adding the internal camera delay, i.e., the JPEG2000 encoding within the camera, the total system delay becomes $1.54 \mathrm{~s}$ versus $1.20 \mathrm{~s}$ for sequential processing. This is also shown in Fig. 13, which shows the trade-off between delay and throughput for a system with 1 to 5 pipeline stages ( 1 pipeline stage refers to sequential processing). To facilitate a faster driving speed of the vehicle, the throughput should be as high as possible, which explains why we have chosen to use a five-stage pipeline. It is our opinion that a $28 \%$ increase in delay is acceptable to achieve a $3.5 \times$ higher throughput in frame rate.

\subsection{GPU Processing}

As discussed in the previous section and shown in Table 2, several time consuming processing tasks are executed on a GPU. Although their implementations lie outside the scope of this work, each GPU task is described briefly.
Image decoding: The image decoding features a thirdparty JPEG2000 decoder on the GPU. This reduces the typical decoding time for a stereo pair from $405 \mathrm{~ms}$ on the CPU to $44 \mathrm{~ms}$ on the GPU.

Rectification and compensate lens vibration: The stereo rectification comes down to applying an inverse transformation that ensures that corresponding points in the left and right stereo images lie on the same scanline. This inverse transformation is performed on the GPU. For the lens-vibration compensation mentioned in Sec. 4.2, BRIEF feature extraction and brute-force feature matching are executed on the GPU. This results in an execution time of $29 \mathrm{~ms}$ on the GPU versus $74 \mathrm{~ms}$ on the CPU.

Disparity estimation: The disparity estimation is a straightforward GPU port of the algorithm described in Sec. 4.2. This typically reduces the execution time of the disparity estimation from $3200 \mathrm{~ms}$ on the CPU to $132 \mathrm{~ms}$ on the GPU.

Simulate the live viewpoint: The viewpoint synthetization from Sec. 4.4 is performed using OpenGL. The same holds for the disparity warping, which involves the same procedure, where texture is replaced by the disparity map. The latter includes the application of the flow fields, estimated during registration refinement of the texture map.

Registration refinement: This is the pixel-accurate registration refinement that is part of the 2.5-D hierarchical alignment. ${ }^{20}$ This refinement involves a dense patchbased correlation that is similar to computing a dense flow map. The resulting flow map is used to correct for minor misregistrations.

\subsection{Off-Line Preprocessing}

The complexity of some of the processing tasks is high, while their output only needs to be available for the historical data, i.e., for images recorded during a previous patrol. To speed up the live processing chain, those tasks have been moved to a separate off-line preprocessing pipeline (Fig. 3) that is executed after a patrol is finished. The live

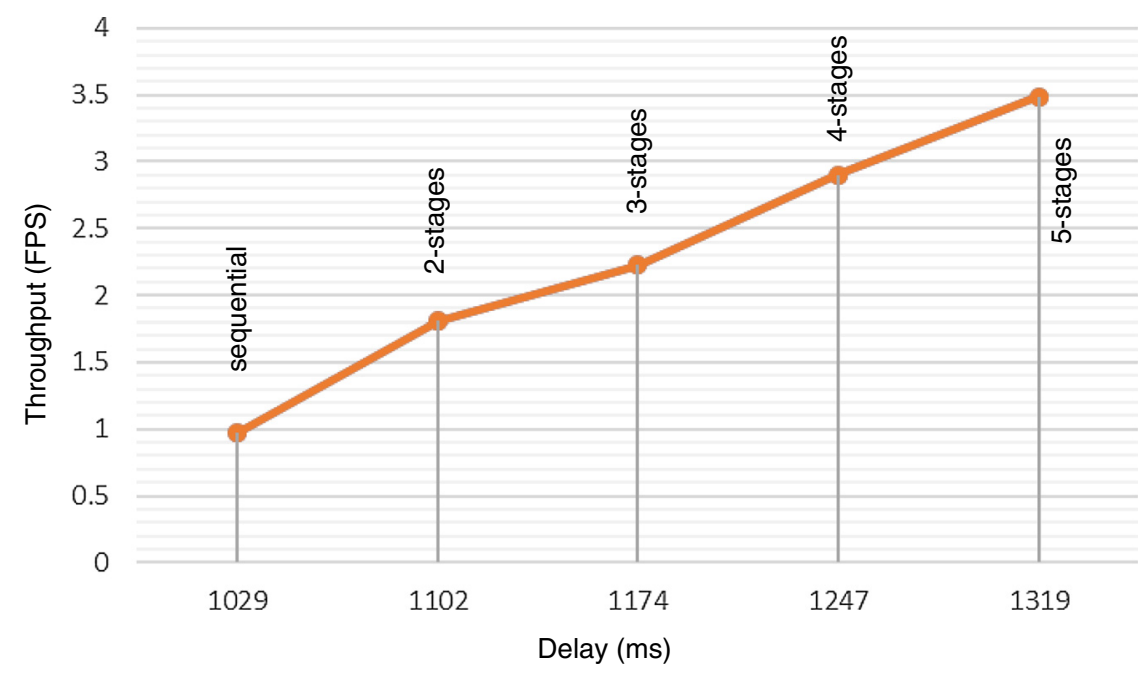

Fig. 13 Throughput versus delay for a variable number of pipeline stages. 
Table 3 Off-line preprocessing algorithms distributed over the different off-line pipeline stages.

\begin{tabular}{llcc} 
Stage & \multicolumn{1}{c}{ Processing task } & Time $(\mathrm{ms})$ & CPU/GPU \\
\hline $\mathbf{1}$ & Image decoding & 43 & GPU \\
& $\begin{array}{l}\text { Rectification and } \\
\text { compensate lens vibration }\end{array}$ & 37 & Both \\
& Compute features & 19 & Both \\
\cline { 3 - 3 } & & 99 & \\
$\mathbf{3}$ & Bilateral disparity filter & 196 & GPU \\
$\mathbf{4}$ & Ground surface mesh & 160 & CPU \\
\hline
\end{tabular}

processing can then simply load the precomputed data whenever necessary.

Similar to the live processing, the off-line preprocessing tasks can be pipelined to increase the throughput of the system, as shown in Table 3. This results in a throughput of $\sim 5$ frames per second (fps). This means that for each hour of live processing, we need 35 min of off-line processing, which is typically performed overnight.

Since the lens-vibration compensation is computed during off-line processing, the related task in stage 3 of the live processing (Table 2) does not need to recompute this data for the historical frames. Therefore, execution time is lower than that of the same task in stage 1 .

\section{Experiments and Results}

For the experiments in this work, the change detection system was mounted on a vehicle as shown in Fig. 1. First, videos of the test environment were recorded prior to the placement of test objects, which resulted in the historical images. Next, several changes were made to the environment, such as the placement of physical objects and digging tracks on the road surface. Finally, the live videos were acquired after the changes were planted.

Experiments are twofold. First, system characteristics are evaluated based on simplified tests, i.e., designed to specifically evaluate a certain aspect of the system. Finally, a more realistic test is performed where objects with a similar contrast and volume as actual IED indicators have been placed alongside a 300-m test track, both on the road surface and in trees. Moreover, all planted changes have been geo-referenced with an accurate GPS location, achieving an accuracy of $10 \mathrm{~cm}$. This enables the evaluation of the detection accuracy of the system at different distances to each object. The results of each experiment are discussed individually, where all experiments feature stereo images of $1920 \times 1440$ pixels and a stereo baseline of $60 \mathrm{~cm}$.

\subsection{Validation Criteria}

Performance criteria are based on the amount of true positives (TP), false positives (FP), and false negatives (FN). Here, a TP is a correctly detected change, an FP is a false alarm, and an FN is a missed change, meaning that the system did not find the change. We consider the Jaccard index as a scoring metric, where a detected change is considered a TP if the Jaccard index exceeds a predefined threshold: $J(A, B)=(A \cap B) /(A \cup B)>T$ and is an FP otherwise. Parameter $B$ represents the rectangular bounding box containing the detected change and $A$ denotes the annotated rectangular bounding box of a planted change. The threshold is empirically set to $T=0.1$ for all our experiments. This ensures that a partially detected object, which is sufficient warning for an operator, is not counted as an FN. This is a valid assumption within the targeted use case of early warning, where the exact segmentation of the threat is not required, as long as the threat itself is detected.

From the TP, FP, and FN counts, the recall $R$ of the system is calculated by $R=\mathrm{TP} /(\mathrm{TP}+\mathrm{FN})$, where the recall stands for the fraction of correctly detected changes from the set of all planted changes. The false alarm rate is defined as the number of FP divided by the total number of evaluated frames.

In our experiments, object contrast refers to the contrast between a change and its surrounding background. To clearly evaluate the contrast of an object with respect to the scene, we use the following metric:

$$
C=\frac{\sum_{b \in B}\left(b_{R}-\overline{x_{R}}\right)^{2}+\sum_{b \in B}\left(b_{G}-\overline{x_{G}}\right)^{2}+\sum_{b \in B}\left(b_{B}-\overline{x_{B}}\right)^{2}}{\sum_{b \in B}\left(b_{R}-\overline{b_{R}}\right)^{2}+\sum_{b \in B}\left(b_{G}-\overline{b_{G}}\right)^{2}+\sum_{b \in B}\left(b_{B}-\overline{b_{B}}\right)^{2}},
$$

where $C$ denotes the contrast ratio, $B$ represents a (background) area around the object, $\overline{x_{R}}, \overline{x_{G}}, \overline{x_{B}}$ refer to the average red, green, and blue values of the object. The $b_{R}, b_{G}, b_{B}$ denote the red, green, and blue values of the background pixels and $\overline{b_{R}}, \overline{b_{G}}, \overline{b_{B}}$ the average background colors, respectively.

Using Eq. (5), we define three different contrast levels:

- High contrast: $C>12$,

- Medium contrast: $5 \leq C<12$,

- Low contrast: $C<5$,

where it should be noted that a contrast factor of unity is the absolute minimum, i.e., both the object and background have the exact same RGB value everywhere. In practice, noise will always yield higher contrast factors, hence the low-contrast factor threshold was empirically set to 5. At this setting, the object and background have almost no visual contrast.

Throughout this paper, recall values are based on all detected and missed planted changes up to 40-m distance from the vehicle.

\subsection{Contrast Test}

This test features test objects of $9 \times 18 \times 9 \mathrm{~cm}$ with varying contrast against the background. Table 4 shows the recall for the different contrast types defined in Sec. 6.1, where the recall is based on all video frames, starting at a distance of $40 \mathrm{~m}$ from the vehicle. The high-contrast objects are detected in nearly every frame, while the medium-contrast objects are detected in the majority of frames. Objects with low contrast to the background are not detected reliably, 
Table 4 Recall per contrast type.

\begin{tabular}{llcc}
\multicolumn{2}{l}{ Recall per contrast } & Average \\
\hline High & Med & Low & False alarms \\
0.89 & 0.56 & 0.03 & 0.04 \\
\hline
\end{tabular}

without increasing the false alarm rate. This was expected considering their poor contrast, of which an example is shown in Fig. 14. The false alarm rate during this experiment was 0.04 , meaning that a single false detection appeared every 25 frames.

\subsection{Lateral Object Emplacement Test}

For this test, test objects of $9 \times 18 \times 9 \mathrm{~cm}$ have been placed at varying lateral displacements into the side terrain, i.e., distance offsets perpendicular to the driving direction. Table 5 shows the recall for a single high-contrast object at different lateral displacements. We note that the recall is only slightly affected, which is caused by the object leaving the FoV of the second camera at an earlier stage for large lateral displacements. Although the table shows results for a single object, the same trend was observed for the other test objects.

\subsection{Trajectory Deviation Test}

In this test, the effect of a different driving trajectory on the detection performance is evaluated. For this purpose, a single historical video was recorded while driving at the right side of the road, after which objects were placed on the ground surface alongside the road. After test-object placement, six different "live" videos were acquired with different lateral displacements with respect to the historical driving trajectory. The chosen lateral displacements are 0, 1.4, 2.8, 4.2, 5.6 , and $7 \mathrm{~m}$ in between the parallel trajectories. This setup leads to increasing viewpoint differences between the live and historical images.

In addition to the $9 \times 18 \times 9 \mathrm{~cm}$ blocks used in the previous tests (Table 5), this test also features smaller and hence more challenging test objects. Table 6 shows the recall and false alarm rate of the system for different lateral displacements between the live and historical driving trajectory. Although the recall of high-contrast objects slightly degrades for large viewpoint differences, the system shows an overall
Table 5 Recall for the same high-contrast object at different lateral displacements into the side terrain. Recall is based on all detections up to $40 \mathrm{~m}$ from the vehicle.

\begin{tabular}{lccccc} 
Object displacement & $0 \mathrm{~m}$ & $3 \mathrm{~m}$ & $6 \mathrm{~m}$ & $9 \mathrm{~m}$ & $12 \mathrm{~m}$ \\
\hline Recall & 0.92 & 0.97 & 0.94 & 0.91 & 0.85 \\
\hline
\end{tabular}

Table 6 Recall of the change detection system for different lateral driving trajectories, e.g., when driving parallel to the reference trajectory at an offset of 0 to $7 \mathrm{~m}$. This test is based on a more challenging environment than the test of Table 5.

\begin{tabular}{lcccccc}
\hline Displacement $(\mathrm{m})$ & 0 & 1.4 & 2.8 & 4.2 & 5.6 & 7 \\
\hline Recall for & & & & & & \\
High contrast & 0.80 & 0.87 & 0.73 & 0.72 & 0.71 & 0.62 \\
Med contrast & 0.47 & 0.54 & 0.51 & 0.60 & 0.62 & 0.68 \\
Low contrast & 0.26 & 0.37 & 0.33 & 0.33 & 0.34 & 0.21 \\
False alarms & 0.08 & 0.11 & 0.17 & 0.22 & 0.24 & 0.22 \\
\hline
\end{tabular}

robustness to such displacements, where objects are still found in the majority of all frames.

The reader may notice an increase in recall for the medium-contrast objects for larger displacements. Further analysis has shown that the lateral displacement modifies the visible background around each object (parallax). In this case, the contrast of the medium objects to their backgrounds slightly improved, while still satisfying the medium-contrast constraints from Sec. 6.1.

The false alarm rate does increase with larger lateral displacements. This is expected, since the viewpoint synthesis applied during registration may exhibit minor rendering artifacts for large viewpoint differences, such as blurring.

\subsection{Realistic Test}

This test involves the use of a broader variety of test objects, including digging tracks, a tree trunk, $0.5-1$ soda cans on, and above the ground surface as well as $9 \times 18 \times 9 \mathrm{~cm}$ blocks in various colors placed in the side terrain. In contrast to the

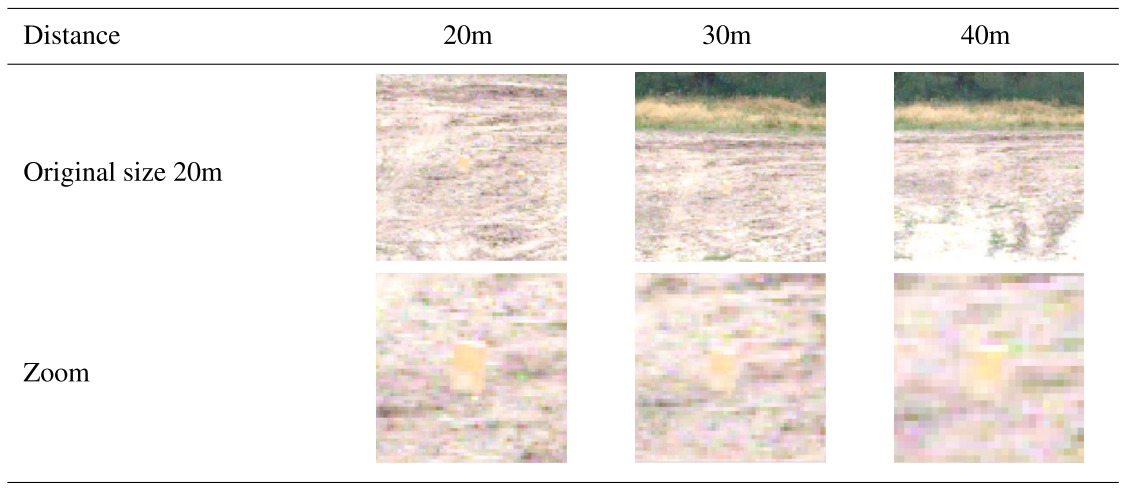

Fig. 14 Example of a low-contrast object at several distances from the camera, where the top row shows the original resolution and the bottom row portrays a zoomed version of the same object. 


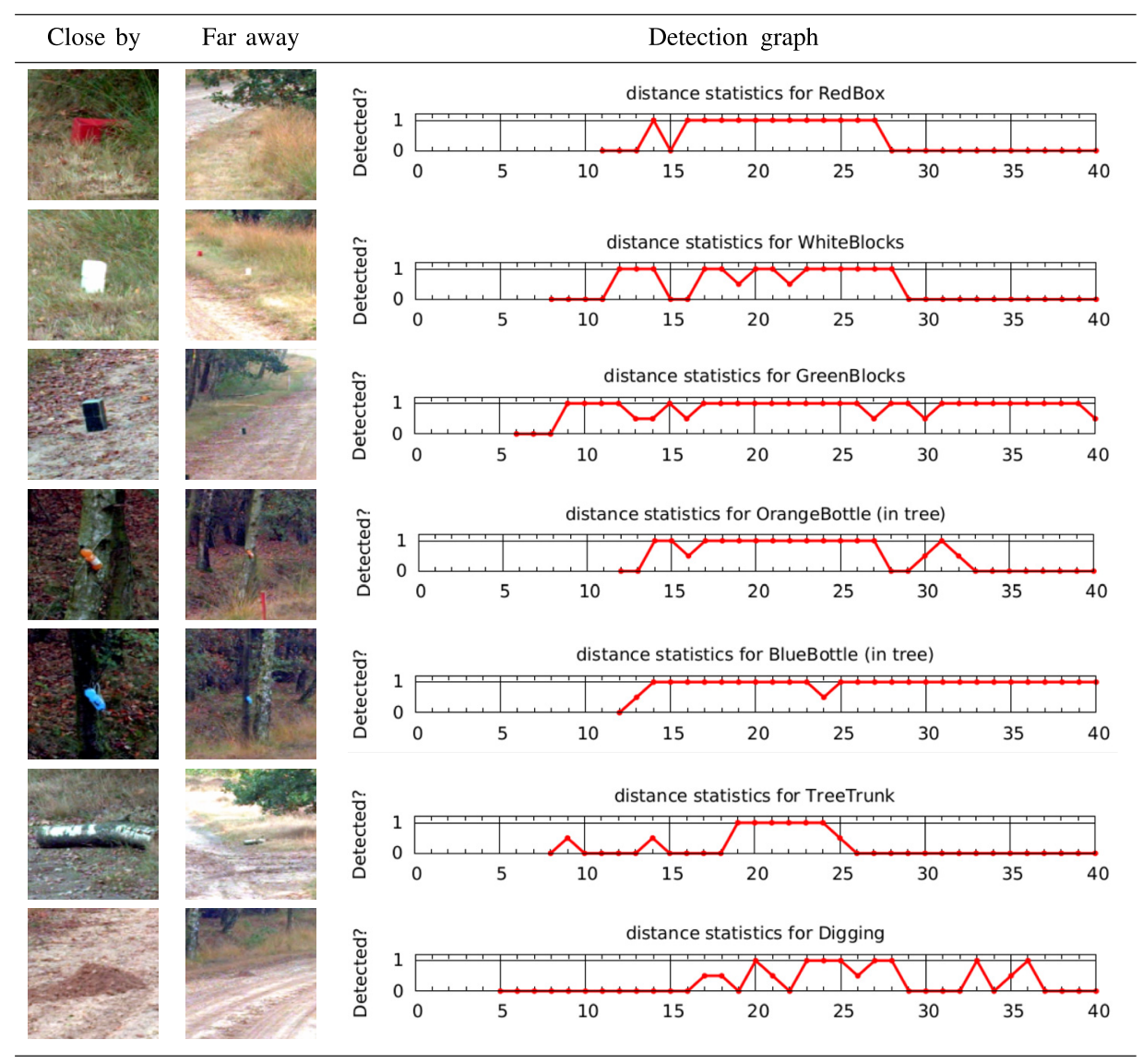

Fig. 15 Examples of test objects used in the realistic test and their corresponding detection graphs versus the detection distance. In these graphs, a "1" means the object is detected, while a "0" means it was missed at that specific distance. As the system captures images every $0.5 \mathrm{~m}$, each bin in the graph corresponds to two images. Therefore, the value "0.5" in the graph means that it was detected in one of the two images.

previous tests, the objects are placed at less obvious locations and are sometimes even partially occluded, as can be seen in Fig. 15. Next to the variety in test objects, the historical and live video are captured under different weather conditions, where the first was recorded with an overcast sky and the latter during sunshine, causing challenging shadows (Fig. 16). Moreover, the driver was asked to drive the same route multiple times without explicit instruction on following the exact same trajectory. We argue that this experiment better resembles a realistic operational scenario, where indicators of an IED have been placed along the route of a patrol and weather conditions between the two patrols are different.

Figure 15 shows the detection scores for a subset of the test objects. The detection graphs show at which distance an object was properly detected. All high-contrast objects within the FoV of the camera were already detected at 40-m distance. The medium-contrast and partially occluded objects are also stably detected over time, although at a

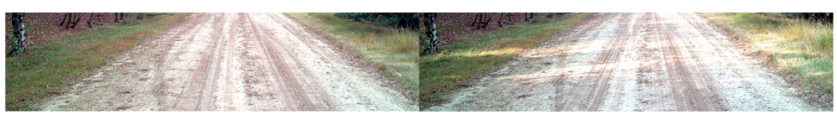

Fig. 16 Different lighting conditions during the realistic test, where the system has to cope with shadows. somewhat shorter distance to the vehicle. Although the digging marks have no depth cues and their texture strongly resemble the background texture, they are still detected by the system. It should be noted that once an object has been detected in multiple frames, it can be tracked in consecutive frames and shown to the operator as a possible threat, even though the system may no longer detect it at close proximity. However, for evaluation purposes, Fig. 15 shows the actual detections and does not take into account the possibility of tracking objects after their detection.

\section{Discussion}

Taking into account the delay of the proposed change detection system, Sec. 2 shows that threats should be detected at 33-m distance from the vehicle, to facilitate stopping the vehicle at a safe stand-off distance. From the results presented in Sec. 6, we confirm that high-contrast changes with a size of $9 \times 18 \times 9 \mathrm{~cm}$ are reliably detected at even earlier distances. Smaller high-contrast changes as well as mediumcontrast changes are also found, although they are typically first detected at a distance of $\sim 25$ to $30 \mathrm{~m}$. This means that the operator has less time to make his decision on the threat, compared to the decision time shown in Table 1. However, as this work is meant as a stepping stone toward an unmanned ground vehicle (UGV), the UGV could slow down after an initial threat detection by the system. This will give the 
operator additional time to analyze the threat prior to the moment that the threat comes within the stand-off distance.

To improve the detection rate of low-contrast test objects and objects within shadow areas, additional preprocessing in the form of contrast enhancement could be applied to better detect such objects. Although promising results are obtainted by, e.g., Huang et al., ${ }^{27}$ their approach does not execute in real-time. As an alternative, contrast limited adaptive histogram equalization $\left(\mathrm{CLAHE}^{28}\right)$ could be employed, which does operate in real-time. However, preliminary experiments with CLAHE in the proposed system yield a significant increase in false alarms. This is most likely caused by a different enhancement for the live and historical images, causing additional visual changes. Future work could look into contrast enhancement techniques that also consider equalization between the live and historical images. For example, by adapting the work of Huang et al. ${ }^{27}$ to take into account both the live and the historical images and the real-time requirement.

Temporal consistency of changes is currently only evaluated over two successive frames. Considering that false alarms are typically less stable over time, the temporal range could be increased to suppress such false alarms. This yields a trade-off between the true-positive rate and the false-alarm rate. Increasing the temporal range enables the system to build up more confidence prior to giving an alarm, thereby reducing the amount of false alarms. However, this also means that changes are detected at a later time, reducing the true-positive rate of the system. As a result, the operator has less time to react to a possible threat. In this work, we choose to maximize the stand-off distance of the system, i.e., to minimize the detection delay by restricting the temporal range to two images. After future optimizations of the system throughput, thus when increasing the temporal range has less impact on the system delay, this trade-off may be reconsidered.

\section{Conclusions}

We have proposed a complete change detection system to be used as an early warning system for IED, where analysis is no longer limited to the ground surface. This real-time system consists of a high-end stereo camera, combined with an image-analysis processing system that performs automatic change detection during patrols. Changes are found by comparing the live image to a historical image of the same scene acquired during a previous patrol, where the image selection is based primarily on the GPS location and camera orientation. Next, the 3-D scene geometry acquired through disparity estimation is used to build a textured 3-D model of the historical scene, which is used to synthesize the historical scene as if viewed by the live camera. An initial set of changes is found in the CIE-Lab space, where the color (AB) and brightness (L) channel(s) are processed independently. False changes are rejected by applying a set of spatiotemporal filters, resulting in a limited set of confident changes to be presented to the operator through an interactive GUI.

With respect to performance, the change detection system shows promising results, where high-contrast objects are detected in nearly every image and medium-contrast objects in the majority of all images. More specifically, the achieved throughput of $3.5 \mathrm{fps}$ is considered sufficient for the operator to analyze the threat in real time. During the validation tests, we have encountered approximately one unique false alarm per $20 \mathrm{~s}$. We argue that this number will be reduced by the foreseen multisensor verification. Moreover, it is demonstrated that the system is robust to both lateral displacements of the vehicle and to different object placements.

We have presented a real-time design of this complex system, where both GPUs and a five-stage pipeline construction have been employed to enable real-time operation using commodity hardware. At present, the key for obtaining real-time performance resides in a clever separation of on-line and off-line processing tasks. In the near future, improvements in both CPU and GPU will enable to reconsider this separation and increase the frame rate of the system.

Although we recommend extending the system with additional sensor modalities to enable the detection of lowcontrast objects and further reduce the false alarm rate, the proposed change detection system shows already a feasible detection performance in the visual spectrum. In our opinion, the proposed system forms a good basis for early detection of (indicators of) IED from an UGV.

\section{Acknowledgments}

This work has been supported by the "Defence Expertise Centre Counter-IED" (DEC C-IED) of the Netherlands Ministry of Defence and has been founded through the National Technology Program (NTP) "Change Detection 2.0."

\section{References}

1. C. Abeynayake and M. D. Tran, "Ground penetrating radar applications in buried improvised explosive device detection," in Int. Conf. Electromagn. Adv. Appl. (ICEAA), pp. 564-567 (2016).

2. H. W. L. Naus, "Modeling of buried wire detection by radio-frequency electromagnetic waves," IEEE Geosci. Remote Sens. Lett. 10, 160-164 (2013).

3. DefenceIndustryReports, "IEDS-learning from history," http://www .defenceindustryreports.com/ieds_learning_from_history.html (2 November 2017).

4. J. B. Sigman et al., "High-frequency electromagnetic induction sensing of nonmetallic materials," IEEE Trans. Geosci. Remote Sens. 55(9), 5254-5263 (2017).

5. D. W. J. M. van de Wouw et al., "Real-time change detection for countering improvised explosive devices," Proc. SPIE 9026, 90260 T (2014).

6. STO, "Ground vehicle based change detection," Technical Report (2014).

7. R. J. Radke et al., "Image change detection algorithms: a systematic survey," IEEE Trans. Image Process. 14, 294-307 (2005).

8. V. Venkateswaran et al., "A survey on unsupervised change detection algorithms," in Int. Conf. Circuits, Power and Comput. Technol. (ICCPCT), pp. 897-903 (2013).

9. Changedetection.NET (CDNET), "A video database for testing change detection systems," www.changedetection.net (January 2019).

10. N. Goyette et al., "Changedetection.net: a new change detection benchmark dataset," in IEEE Comput. Soc. Conf. Comput. Vision and Pattern Recognit. Workshops, pp. 1-8 (2012).

11. P.-L. St-Charles, G.-A. Bilodeau, and R. Bergevin, "SuBSENSE: a universal change detection method with local adaptive sensitivity," IEEE Trans. Image Process. 24, 359-373 (2015).

12. R. Qin, J. Tian, and P. Reinartz, "3D change detection-approaches and applications," ISPRS J. Photogramm. Remote Sens. 122, 41-56 (2016).

13. D. Ali-Sisto and P. Packalen, "Comparison of 3D point clouds from aerial stereo images and lidar for forest change detection," Int. Arch. Photogramm. Remote Sens. Spatial Inf. Sci. XLII-3/W3, 1-5 (2017).

14. M. Bláha et al., "Large-scale semantic 3D reconstruction: an adaptive multi-resolution model for multi-class volumetric labeling," in IEEE Conf. Comput. Vision and Pattern Recognit. (CVPR), pp. 3176-3184 (2016).

15. W. Xiao et al., "Street environment change detection from mobile laser scanning point clouds," ISPRS J. Photogramm. Remote Sens. 107, 38-49 (2015). 
16. A. Taneja, L. Ballan, and M. Pollefeys, "Geometric change detection in urban environments using images," IEEE Trans. Pattern Anal. Mach. Intell. 37, 2193-2206 (2015).

17. K. Zhou et al., "3D building change detection between current VHR images and past lidar data," Int. Arch. Photogramm. Remote Sens. Spatial Inf. Sci. XLII-2, 1229-1235 (2018).

18. H. Haberdar and S. K. Shah, "Disparity map refinement for video based scene change detection using a mobile stereo camera platform," in 20th Int. Conf. Pattern Recognit., pp. 3890-3893 (2010).

19. N. Morales, J. T. Toledo, and L. Acosta, "Object detection in nonstationary video surveillance for an autonomous vehicle," in 11th Int Conf. Intell. Syst. Des. and Appl., pp. 690-695 (2011).

20. D. W. J. M. van de Wouw, G. Dubbelman, and P. H. N. de With, "Hierarchical 2.5-D scene alignment for change detection with large viewpoint differences," IEEE Rob. Autom. Lett. 1, 361-368 (2016).

21. D. W. J. M. van de Wouw et al., "Fast 3D scene alignment with stereo images using a stixel-based 3D model," in Int. Conf. Comput. Vision Theory and Appl. (VISAPP), Vol. 4, pp. 250-259 (2018).

22. P. F. Alcantarilla et al., "Street-view change detection with deconvolutional networks," Auton. Rob. 42, 1301-1322 (2018).

23. B. Ranft and T. Strauß, "Modeling arbitrarily oriented slanted planes for efficient stereo vision based on block matching," in 17th Int. IEEE Conf Intell. Transp. Syst. (ITSC), pp. 1941-1947 (2014).

24. D. W. J. M. van de Wouw et al., "Real-time estimation of the 3D transformation between images with large viewpoint differences in cluttered environments," Electron. Imaging 2017(13), 109-116 (2017).

25. A. Woodland and F. Labrosse, "On the separation of luminance from colour in images," in Int. Conf. Vision, Video and Graphics, pp. 29-36 (2005).

26. Q. Yang, L. Wang, and N. Ahuja, "A constant-space belief propagation algorithm for stereo matching," in IEEE Comput. Soc. Conf. Comput. Vision and Pattern Recognit., pp. 1458-1465 (2010).
27. Z. Huang et al., "Framelet regularization for uneven intensity correction of color images with illumination and reflectance estimation," Neurocomputing 314, 154-168 (2018).

28. K. Zuiderveld, "Contrast limited adaptive histogram equalization," in Graphics Gems IV, pp. 474-485 (1994).

Dennis W. J. M. van de Wouw received his MSc degree from the Eindhoven University of Technology in 2011. As the change detection system architect at ViNotion, he is now finalizing his PhD thesis on depth-based extensions for change detection, in close collaboration with TU/e.

Frank B. ter Haar is a scientific researcher at TNO Defence, Security and Safety in the Netherlands, with an expertise in 3-D geometric processing, 3-D acquisition, reconstruction, and modeling.

Gijs Dubbelman is an assistant professor with the Eindhoven University of Technology and is heading the mobile perception systems (MPS) research cluster, which focuses on signal processing technologies that allow mobile sensor platforms to perceive the world around them.

Peter H. N. de With is a full professor of the Video Coding and Architectures Group in the Department of Electrical Engineering at Eindhoven University of Technology. He is an IEEE fellow, has (co-)authored over 400 papers on video coding, analysis, architectures, and 3-D processing and has received multiple papers awards $\mathrm{He}$ is a program committee member of the IEEE CES and ICIP and holds some 30 patents. 